\title{
Digital Health Europe (DHE) Twinning on severe asthma-kick-off meeting report
}

\author{
Jean Bousquet ${ }^{1,2,3}$, Anna Bedbrook ${ }^{3,4,5}$, Wienczyslawa Czarlewski ${ }^{4,5,6}$, Giuseppe De Carlo ${ }^{7}$ \\ Joao A. Fonseca ${ }^{8,9}$, Miguel A. González Ballester ${ }^{10}$, Maddalena Illario ${ }^{11}$, Seppo Koskinen ${ }^{12}$, \\ Tiina Laatikainen ${ }^{12}$, Gabrielle L. Onorato ${ }^{3}$, Susanna Palkonen ${ }^{7}$, Vincenzo Patella ${ }^{13}$, Nhân Pham-Thi ${ }^{14}$, \\ Francesca Puggioni ${ }^{15,16}$, Maria Teresa Ventura ${ }^{17}$, Guy Joos ${ }^{18}$, Piotr Kuna ${ }^{19}$, Renaud Louis ${ }^{20}$, \\ Michael Makris ${ }^{21}$, Petra Zalud ${ }^{22}$, Torsten Zuberbier ${ }^{1}$, Claus Bachert ${ }^{23,24,25,26}$, Luisa Brussino ${ }^{27}$, \\ Pedro Carreiro-Martins ${ }^{28,29}$, Carme Carrion y Ribas ${ }^{30}$, Maciej Chalubinski ${ }^{31}$, Elisio M. Costa ${ }^{32}$, \\ Govert de Vries $^{33}$, Bilun Gemicioglu ${ }^{34}$, Dimitra Gennimata ${ }^{35}$, Yann Micheli ${ }^{36}$, Marek Niedoszytko ${ }^{37}$, \\ Frederico S. Regateiro ${ }^{38,39}$, Jan Romantowski ${ }^{37}$, Luis Taborda-Barata ${ }^{40,41}$, Sanna Toppila-Salmi ${ }^{42}$, \\ Ioanna Tsiligianni ${ }^{43,44}$, Frederic Viart ${ }^{45}$, Daniel Laune ${ }^{36}$
}

${ }^{1}$ Charité Universitätsmedizin Berlin, Humboldt-Universität zu Berlin, and Berlin Institute of Health, Comprehensive Allergy Center, Department of Dermatology and Allergy, Berlin, Germany; ${ }^{2}$ University Hospital Montpellier, Montpellier, France; ${ }^{3}$ Maladies Chroniques pour un Viellissement Actif, (Macvia-France), Montpellier, France; ${ }^{4}$ Allergic Rhinitis and its Impact on Asthma (ARIA), Montpellier, France; ${ }^{5}$ Mobile Airways Sentinel nekworK (MASK-air), Montpellier, France; ${ }^{6}$ Medical Consulting Czarlewski, Levallois, France; ${ }^{7}$ European Federation of Allergy and Airways Diseases Patients' Associations, Brussels, Belgium; ${ }^{8}$ Center for Research in Health Technology and Information Systems, Faculdade de Medicina da Universidade do Porto, Porto, Portugal; ${ }^{9}$ Medida, Lda Porto, Portugal; ${ }^{10}$ Department of Information and Communication Technologies, Universitat Pompeu Fabra, Barcelona, Spain, ICREA, Barcelona, Spain; ${ }^{11}$ Division for Health Innovation, Campania Region and Federico II University Hospital Naples (R\&D Unit and Department of Public Health), Naples, Italy; ${ }^{12}$ Finnish Institute for Health and Welfare, Helsinki, Finland; ${ }^{13}$ Division of Allergy and Clinical Immunology, Department of Medicine, Agency of Health ASL Salerno, "Santa Maria della Speranza" Hospital, Battipaglia, Salerno, Italy; ${ }^{14}$ Ecole Polytechnique Palaiseau, IRBA (Institut de Recherche bio-Médicale des Armées), Bretigny, France; ${ }^{15}$ Personalized Medicine Clinic Asthma \& Allergy, Humanitas Clinical and Research Center IRCCS, Rozzano, MI, Italy; ${ }^{16}$ Department of Biomedical Sciences, Humanitas University, Pieve Emanuele, MI, Italy; ${ }^{17}$ University of Bari Medical School, Unit of Geriatric Immunoallergology, Bari, Italy; ${ }^{18}$ Department of Respiratory Medicine, Ghent University Hospital, Ghent, Belgium; ${ }^{19}$ Division of Internal Medicine, Asthma and Allergy, Barlicki University Hospital, Medical University of Lodz, Lodz, Poland; ${ }^{20}$ Department of Pulmonary Medicine, CHU Sart-Tilman, and GIGA I3 Research Group, Liege, Belgium; ${ }^{21}$ Allergy Unit "D Kalogeromitros", 2nd Department of Dermatology and Venereology, National \& Kapodistrian University of Athens, "Attikon” University Hospital, Athens, Greece; ${ }^{22}$ tp21 GmbH, Berlin, Germany; ${ }^{23}$ Upper Airways Research Laboratory, ENT Department, Ghent University Hospital, Ghent, Belgium; ${ }^{24}$ International Airway Research Center, First Affiliated Hospital, Sun Yat-sen University, Guangzou, China; ${ }^{25}$ Division of ENT Diseases, CLINTEC, Karolinska Institutet, Stockholm, Sweden; ${ }^{26}$ Department of ENT Diseases, Karolinska University Hospital, Stockholm, Sweden; ${ }^{27}$ Department of Medical Sciences, Allergy and Clinical Immunology Unit, University of Torino \& Mauriziano Hospital, Torino, Italy; ${ }^{28}$ Serviço de Imunoalergologia, Hospital de Dona Estefânia, Centro Hospitalar de Lisboa Central, Lisbon, Portugal; ${ }^{29} \mathrm{CEDOC}$, Faculdade de Ciências Médicas (FCM), Universidade Nova de Lisboa, Lisbon, Portugal; ${ }^{30}$ School of Health Sciences and UOC eHealth Center, Universitat Oberta de Catalunya, Barcelona, Spain; ${ }^{31}$ Department of Immunology and Allergy, Medical University of Lodz, Lodz, Poland; ${ }^{32}$ Faculty of Pharmacy and Competence Center on Active and Healthy Ageing of University of Porto (Porto4Ageing), Porto, Portugal; ${ }^{33}$ Peercode BV, Geldermalsen, The Netherlands; ${ }^{34}$ Department of Pulmonary Diseases, Istanbul University-Cerrahpasa, Cerrahpasa Faculty of Medicine, Istanbul, Turkey; ${ }^{35}$ Department of Pharmacy, Athens General Hospital "Korgialenio-Benakio" Hellenic Red Cross, Athens, Greece; ${ }^{36}$ KYomed INNOV, Montpellier, France; ${ }^{37}$ Medical University of Gdańsk, Department of Allergology, Gdańsk, Poland; ${ }^{38}$ Allergy and Clinical Immunology Unit, Centro Hospitalar e Universitário de Coimbra, Coimbra, Portugal; ${ }^{39}$ Institute of Immunology, Faculty of Medicine, University of Coimbra, Coimbra, Portugal; ${ }^{40}$ Health Sciences, University of Beira Interior, Covilhã, Portugal; ${ }^{41}$ Department of Immunoallergology, Cova da Beira University Hospital Centre, Covilhã, Portugal; ${ }^{42}$ Skin and Allergy Hospital, Helsinki University Hospital and University of Helsinki, Helsinki, Finland; ${ }^{43}$ Health Planning Unit, Department of Social Medicine, Faculty of Medicine, University of Crete, Crete, Greece; ${ }^{44}$ International Primary Care Respiratory Group IPCRG, Aberdeen, Scotland; ${ }^{45}$ Advanced Solutions Accelerator, Clapiers, France

Correspondence to: Professor Jean Bousquet. 273 avenue d'Occitanie, 34090 Montpellier, France. Email: jean.bousquet@orange.fr. 
Submitted Jan 13, 2021. Accepted for publication Apr 09, 2021.

doi: $10.21037 /$ jtd-21-792

View this article at: http://dx.doi.org/10.21037/jtd-21-792

\section{Introduction}

Mobile Airways Sentinel NetworK (MASK) is a Good Practice of DG Santé on digital tools for citizen empowerment and person-centred care for rhinitis and asthma multimorbidity. It was presented in the Joint Research Centre of Ispra (December 15, 2018) (1-5).

MASK is based on:

* An App for rhinitis and asthma developed by the MACVIA-France EIP on AHA reference site (Allergy Diary, now MASK-air ${ }^{\circledR}$ ), available in 27 countries and fully validated (1);

* An interoperable electronic clinical decision support system (CDSS) (6);

* An interoperable web-based physician's questionnaire (7) and

* An EIT Health project POLLAR (Impact of air POLLution on Asthma and Rhinitis) which predicts alerts for pollen and pollution peaks (8).

MASK has led to:

* A better understanding of rhinitis in terms of phenotypes and management $(1,9)$;

* A better understanding of the links between allergy and pollution $(10,11)$;

* A proof-of-concept on the digital transformation of health and care to sustain Planetary Health (12);

* Clinical trials $(13,14)$.

MASK is embedded in the European Union (EU) political agenda (15) and is aimed at strengthening the EU digital single market (DSM) (16).

A first Twinning [success story of Digital Health Europe (DHE)] was devoted to rhinitis and asthma. It included 22 reference sites and 22 countries (8). Over 1,000 patients were enrolled. The aim of the first Twinning was to transfer innovation from the MASK-air ${ }^{\circledR}$ App to other reference sites on rhinitis across the life cycle. The analyses of the data using artificial intelligence are underway.

Severe asthma (SA) is an important global health problem leading to exacerbations, deaths and resource utilization. Over $80 \%$ of direct and indirect asthma costs are incurred by SA. Its phenotype and treatment in old age adults are poorly understood. Less than $5 \%$ of asthmatic patients have SA and only collaborative studies can have a sufficient number of participants to perform pooled analyses in order to better phenotype and manage the disease, thanks to the large scale-up allowed by validated digital solutions.

Building on the experience of the first Twinning, (8) the DHE Twinning on SA was planned to form a European network of experts on SA. The kick-off meeting of the DHE Twinning was organized at the Institut Pasteur, Paris, January 10, 2020. This meeting was jointly sponsored by ARIA and the Reference Site Collaborative Network of the European Innovation Partnership on Active and Healthy Ageing (17). It was a GARD research demonstration project following the two meetings held in the same venue in 2019 $(18,19)$.

The current paper reports the minutes of the meeting.

\section{New concepts of allergic multimorbidity}

Allergic diseases are heterogeneous. Clinically, some patients have allergic rhinitis (AR) alone, whereas others have AR and asthma (with or without other allergic manifestations). Few patients have asthma alone, particularly in childhood. There are common genes in asthma and AR multimorbidity [e.g., IL-33: T2 inflammation (20) and IL-5: eosinophil activation (21)], and specific genes in AR alone (TLR). Moreover, ocular symptoms add further complexity to the allergy phenotypes (22) and to SA (23). By combining big data analyses [MASK (3)], classical epidemiologic studies, in silico analyses, transcriptomics [MeDALL $(21,24,25)]$ and gene sequencing (26), the mechanisms of allergic diseases have been reclassified. In particular, polysensitization and multimorbidity represent the extreme allergic phenotype, starting early in life and with the greatest severity. This is associated with $I L-5$ and $I L-33$ activation. It is classically proposed that rhinitis can lead to asthma. However, the exact phenotype of AR prone to developing asthma is still unclear. It is possible that polysensitized individuals can more commonly develop lower airway symptoms. These considerations should be included in any study on SA to better define phenotypes and severity.

\section{DHE Twinning}

The focus of the Twinning is on local, regional and national health and care providers in Europe that wish to adopt 
innovative solutions which are available for transfer from elsewhere. Applying to the tender opportunities of European calls for Horizon 2020, this provides an opportunity to share the experience with a large number of experts in SA and its different shapes, using MASK-air ${ }^{\circledR}$ data across Europe.

Some of the principal aspects will be proposed to improve the innovative aspect of care by digital medicine on the severe shape of asthma. The innovative study will assess different contributors of SA such as climate change and air pollution (27), psychological aspects as well as the impact of stress on SA (28), and, finally, the adherence (29) and use of devices.

Aims of the Severe Asthma-Twinning: using:

* The expertise of the first Twinning (8);

* The World Health Organization (WHO) classification of SA (30);

* The addition of new questions relevant to SA in the MASK-air ${ }^{\circledR}$ app as well as the web-based physician's questionnaire;

* Previous Twinning members with a high patient inclusion rate and including new members.

The general aim of the DHE Twinning is to form a network that will reduce the burden of SA, particularly in old age people.

The specific objectives are:

(I) To form an international network for SA in old age people globally;

(II) To better understand the phenotype and treatment of SA in old age people with possible differences between countries and gender;

(III) To include the results into the Good Practice on Digital Health for disease stratification and personalized health care. With a vision to better prescribe expensive treatments (biologics) and to follow-up the patients using real-world data analyzed as previously in MASK-air ${ }^{\circledR}$ and with artificial intelligence as a proof-of-concept;

(IV) To be the basis for a further deployment beyond the funding, including a network of centres of excellence (CoE) on SA (ARIACare for severe asthma) through an interdisciplinary collaboration and with the involvement of primary care and patients' organisations.

\section{MASK e-platform for shared decision-making (SDM) in airway diseases}

In SDM, both the patient and the physician contribute to the process, placing the patient at the centre of the medical decision-making (31). mHealth applications and other e-tools support SDM (32). The MASK e-platform for SDM in airway diseases is being developed around MASK to allow a uniform and harmonized assessment of airway diseases. It has already added other validated tools [e.g., the Control of Allergic Rhinitis and Asthma Test (CARAT) (33-35) or Air Quality $(8,11)]$ and will be open to integration of third-party features or functionalities. Privacy and personal data protection are also a core requirement, building on the previous work done for the MASK-air ${ }^{\circledR}$ app $(36,37)$.

The platform will be used for several purposes and will use four scenarios:

* The screening of patients when they use the project's app, either in the waiting room or in the physician's office;

* The initial assessment of the patient for the diagnosis and treatment of allergic disease using a physician's web-based questionnaire and a CDSS;

* The follow-up of the patient using the updated MASK-air ${ }^{\circledR}$ app and additional tools depending on the needs;

* The review of the patient by a healthcare professional after a period of follow-up.

The components of the e-platform will include (I) the MASK-air $^{\otimes}$ app, (II) CARAT, COPD and comorbidities screening questionnaires; (III) an e-CDSS for AR and asthma; (IV) lung function monitoring and personalized behaviour recommendations; (V) the Twinning Physician questionnaire; (VI) INSPIRERS' verified inhaler adherence and gamification (38) and (VII) air quality and pollen information.

\section{ARIACARE CoE}

ARIA (Allergic Rhinitis and its Impact on Asthma) was established 20 years ago and includes over 600 members in over 80 countries (1). ARIA Phase 3 was the development of a Good Practice on the digital transformation of health and care in rhinitis and asthma. In ARIA Phase 4 (change management for airway diseases) $(3,39)$, a network of $\mathrm{CoE}$ is being organized. ARIACARE is part of the GA²LEN CoE and includes UCARE and ADCARE (40). Accreditation follows the UCARE proposals and ARIA members will be able to apply. Other $\mathrm{CoE}$ can also apply to ARIACARE.

ARIACARE-Digital is a novel network whose aim is to implement the digital transformation of health and care in airway diseases. Both members of MASK and others 
can join this network. ARIACARE-Digital has links with GA ${ }^{2} L E N(41)$ but both are independent. Three levels of centres are proposed (regular, gold and platinum) with a review each year. Numerous benefits are expected from this innovation.

\section{Web-based physician's questionnaire}

In order to have a common assessment of the patients, a web-based physician's questionnaire has been developed from the rhinitis questionnaire and is available in 17 languages (8). The rhinitis questionnaire includes CARAT (33-35). The asthma questionnaire includes the questions already available for rhinitis, and questions pertinent to asthma have been added:

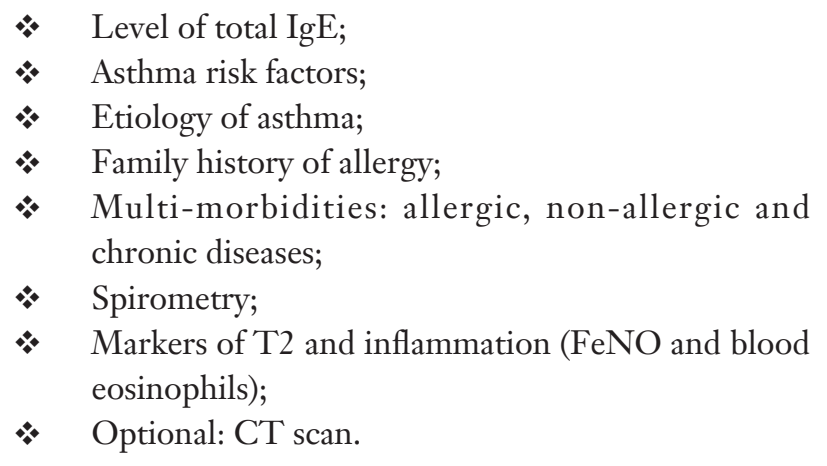

\section{Results of the Twinning rhinitis and asthma}

Over 1,000 patients have been included in the Twinning for rhinitis and asthma (8) and some data have been analyzed (8).

A cross-sectional study on 958 patients with AR in 9 countries used a web-based physician's questionnaire interoperable with the MASK-air ${ }^{\circledR}$ app. Allergists initially recorded the diagnosis of allergy that had been made (rhinitis, rhinoconjunctivitis, current or past asthma) and then used the CARAT questionnaire. Patients were then asked to use the MASK-air ${ }^{\circledR}$ app, and asthma was assessed on the first day of reporting using a visual analogue scale (VAS) and treatments. This is the first study to assess a physician's diagnosis of asthma in AR using electronic realworld data (RWD). The current asthma under-diagnosis was found to be substantial in very experienced and committed allergists. Asthma control was better in CARATdiagnosed asthma than in physician's reported asthma. There is a need for an electronic screening tool to diagnose asthma in rhinitis patients (in preparation).

Another study examined the adherence of patients, and a low adherence was observed for both asthma and rhinitis (in preparation).

\section{The role of patient's organisations in SA}

The European Federation of Allergy and Airways Diseases Patients' Associations (EFA) has identified some of the challenges and needs for SA patients to raise awareness on the impact of SA in patients' lives. First, it is vital to have a timely and accurate diagnosis. Many patients wait for years before knowing their subtype of asthma and getting a tailored and more effective treatment. According to a recent study (42) conducted by EFA, $21 \%$ of people with SA wait for more than 5 years to obtain an accurate diagnosis.

Treatments have improved and new drugs (biologics) are changing the life of patients with SA, despite only a few of them currently undergoing these treatments ( $9 \%$ according to the EFA study). Patient involvement in research is key to improving clinical outcomes, phenotyping SA and taking full advantage of personalized medicine. Patients with SA are more keen to be involved in research compared to patients with moderate or mild asthma, but their involvement is still low ( $77 \%$ have not taken part in research; $44 \%$ are not willing to share their medical data for research).

Finally, the support of family, friends, and other patients can provide motivation in the adherence to treatment. Parents or carers of people with SA need to know how to cope with the disease, and their education is essential for good care. However, in most of the cases (70\%), they do not receive adequate training.

\section{Artificial intelligence and advanced data management}

Recent years have witnessed the resurgence of artificial intelligence and machine learning in many fields of knowledge, not least in health care. Amongst the most promising research directions in the broad field of artificial intelligence, three distinct methodological lines are being explored for their potential use in health studies, including asthma research.

Firstly, deep learning, and particularly deep convolutional neural networks, have proven their value in many applications. Recent methodological developments and the availability of large databases have led to humanlike performance in tasks such as image analysis, including medical imaging (43) and natural language processing (e.g., for the analysis of clinical histories). 
More general machine learning techniques, such as manifold learning, can be used to make sense of large and heterogeneous datasets, such as the ones involved in clinical studies. Examples of these techniques and their application to medical datasets have been shown (44). This strategy seems particularly suited for the multifactorial study of rhinitis and asthma, and is being developed in our on-going research.

Finally, reinforcement learning approaches can be used to simulate populations and optimize policies [or to automatically play games such as Go (45)]. In the field of health care and epidemiology, the combination of these simulations with manifold learning is a very promising line of research.

\section{Registries of SA}

Clinical practice requires a complex interplay between experience and training, research, guidelines and judgement. It must draw not only on data from traditional or classical randomized controlled trials (RCTs), but also from pragmatically designed studies that better reflect reallife clinical practice.

To minimize extraneous variables and to optimize their internal validity, RCTs exclude patients, clinical characteristics and variations in care that could potentially confound outcomes. The result is that respiratory RCTs often enroll a small, non-representative subset of patients and overlook the important interplay and interactions between patients and the real world, which can affect treatment outcomes.

Evidence from real-life studies (pragmatic and observational clinical trials) can be combined with RCT evidence to provide a fuller picture of intervention effectiveness and realistic treatment outcomes.

In developing a real-world evidence (RWE) programme, it is fundamental to distinguish between the sources of RWD and the evidence derived from that data. Evaluating RWE in the context of regulatory decision-making depends not only on the evaluation of the methodologies used to generate the evidence but also on the reliability and relevance of the underlying RWD. These constructs may raise different types of consideration.

Patient registries are a good quality source of RWD that could be used to generate RWE. A patient registry is an organized system that uses observational study methods to collect uniform data (clinical and other) in order to evaluate specified outcomes for a population defined by a particular disease, condition, or exposure and that serves one or more predetermined scientific, clinical, or policy purposes. Their fitness for use in generating RWE requires sufficient processes, such as those to gather follow-up information when needed, to ensure data quality, and to minimize missing or incomplete data.

Regional and/or national SA registries provide valuable country-specific information (46-49). However, they are often limited in scope within the broader definitions of SA, have insufficient statistical power to answer many research questions, lack intra-operability to share lessons learned, and have fundamental differences in data collected, making cross comparisons difficult.

A worldwide registry could bring all SA data together in a cohesive way, under a single umbrella, based on standardized data collection protocols, permitting data to be shared seamlessly. The International Severe Asthma Registry (ISAR) is the first global adult SA registry. It is a joint initiative where national registries retain ownership of their own data but open their borders and share data with ISAR for ethically approved research purposes. Its strength comes from the collection of patient-level, anonymous, longitudinal, real-life, standardized, high-quality data (using a core set of variables) from countries across the world, combined with organizational structure, database experience, inclusivity/openness, and clinical, academic, and database expertise. This provides data with sufficient statistical power to answer important research questions, sufficient data standardization to compare across countries and regions, and the structure and expertise necessary to ensure its continuance and the scientific integrity and clinical applicability of its research.

\section{Country-based RWD: Finland}

Finland has developed a very extensive health system and can be considered as a pioneered country for RWD. Some examples are given in this paper (50-53).

\section{National Register Data}

* Administrative registers in Finland use a unique personal identification number assigned to each resident (since 1964).

- National registers include health-related data on:

- Causes of death;

- Treatment periods and diagnoses (ICD-10) in hospitals; 
Table 1 Prevalence of selected diseases in the population-based sample of the National Health Examination Survey (FinHealth 2017): comparison between two data sources: survey data and registers on the use of hospital and primary care

\begin{tabular}{lccc}
\hline Diseases & Survey & Register & Cohen's Kappa \\
\hline Hypertension & 42.7 & 11.1 & 0.24 \\
Diabetes & 10.1 & 9.1 & 0.85 \\
Asthma & 8.6 & 7.3 & 0.71 \\
Depression & 6.7 & 2.4 & 0.37 \\
\hline
\end{tabular}

- Diagnoses in primary health care arranged by the public sector (ICD-10, ICPC);

- Reimbursed and other prescribed medications;

- Sickness benefits;

- Work disability pensions and the diagnoses;

- Cancer.

Most of the relevant registers are maintained by the Finnish Institute for Health and Welfare (THL), Statistics Finland and the National Social Insurance Institution (KELA).

* The coverage of register data is quite good in selected diseases, but poor in others (Table 1).

\section{Register data in North Karelia}

North Karelia is one of the 20 hospital districts in Finland. It includes 14 municipalities and a population of 163,000 .

* Health and social services are organized by Siun sote which is a joint municipal authority of social and health services in the region;

* There is one central hospital and 22 health stations in the region;

* Since 2010, a joint electronic health record (EHR) has been in use in the region covering both primary and secondary level care;

* EHR is structural in format, enabling easy data retrieval. The database is administered by the North Karelia IT-Center and the Siun sote reporting unit;

* The EHR database includes information on all visits to both primary and specialized care services, stays at wards in health centres or hospitals, clinical procedures and investigations, laboratory tests and prescriptions;

* The coverage of recorded diagnoses on physician visits is almost $100 \%$. All laboratory test results are recorded in the database directly by the laboratory service provider (one accredited organization in the whole region) and results of clinical investigations such as clinico-physiological tests (e.g., spirometry) are recorded in the database in structural format;

* The data are already widely used in analyzing outcomes of care in the region and in developing quality of care reporting systems.

National Health Surveys (https://thl.fi/en/web/thlfi-en/ statistics/information-on-statistics)

These include:

* Interview/questionnaire surveys (HIS), annual since the 1970s;

* Comprehensive health examination surveys (HES) since the 1970s;

- Population-based sample, generally around 10,000 subjects;

- Most HES-surveys include interviews and questionnaires, personal health examinations with physical measurements and blood samples as well as functional capacity tests;

- $\quad$ All HES-surveys include questions on asthma: symptoms, treatment;

- Selected HES-surveys include spirometry (with or without bronchodilatation).

* All health surveys include a register-based followup of deaths, hospital care, drug purchases, entitlements to specially reimbursed medication, sickness benefits, work disability pensions, etc.

\section{DSM and cross-border data exchange}

Current demographic trends challenge the sustainability and equity of European health systems $(54,55)$. A paradigmatic shift from reactive disease management to proactive care, that is developed around a citizen empowered to take part in the health plan, can possibly contribute to achieving a triple win for our Society: improved health outcomes, sustainability and development (56).

Providing equitable public health services that are of high quality within a sustainability framework relies on digital innovation and cross-border interoperability. Therefore, in Europe, providing secure access to genomic and other health data among Member States is essential for better health and care delivery to European citizens. This is also pivotal in ensuring that the EU remains at the forefront of health research (57).

Public health systems throughout the EU have been 
making huge efforts to develop around citizens' needs. They are at the centre of service design, organization and provision. Data-driven healthcare innovation is effective when citizens play an active role in the setup of personalized treatment, respectful of their right to data privacy. In order to overcome data silos, lack of interoperability and fragmentation of initiatives across the $\mathrm{EU}$, enhanced cooperation between member states is essential.

The DSM strategy (58) aims to open up digital opportunities for people and businesses and to enhance Europe's position as a world leader in the digital economy. It provides the possibility of implementing the Digital Transformation of Health and Care by:

* Scaling-up digital innovation through regional ecosystems;

* Encouraging peer-adoptions of reference good practices;

- Promoting stronger connections across regions and cooperation in the field of active and healthy ageing (AHA).

The priorities identified by the DSM strategy are:

(I) Citizens' access to health data: Focusses on citizens' secure access to their health data, also when they are abroad. The goal is to enable citizens to exert their right to access their health data across the $\mathrm{EU}$, including, inter alia, the interoperability of EHR systems;

(II) Personalized medicine through shared European data infrastructure. This stresses the importance of personalized medicine through a shared European data infrastructure;

(III) Citizen empowerment and person-centred care. This empowers people to look after their health, stimulating prevention and enabling feedback and interaction between users and healthcare providers.

In order to achieve the DSM objectives, the European Commission (EC) has been supporting the EIP on AHA since 2013 (59). This horizontal Partnership includes 2 main, interconnected strains:

* The reference sites (17);

* The thematic action groups (60);

The three main pillars of the partnership are:

* The blueprint on the Digital Transformation of Health and Care (61). MASK is an example of this strategy (16);

* The innovation to market strategy;

* A shared monitoring and assessment tool: the

\section{MAFEIP.}

The three pillars have been developed by the collaborative efforts of the partners. Tailored maturity patterns grounded on the exchange of good practices are currently progressing and are being developed by international expert clusters. They are being tailored to locoregional contexts to foster and speed up adoption. The partnership represents a key enabling factor in the scale up of innovative good practices such as MASK, that can mitigate the impact of chronic diseases.

\section{Allergic diseases and asthma in older people}

According to the WHO, the population aged 65 and above is destined to double in number within the next 40 years, and respiratory pathologies are today the main cause of mortality in Europe and the US (62). Among the elderly, rhinitis is one of the most common chronic diseases, affecting $20 \%$ of the patients, with asthma rates ranging between $4 \%$ and $13 \%$.

MASK implements information and communications technology (ICT) mobile technology via a mobile phone app which collects the daily data of VASs for overall allergic symptoms. The aim is to ensure an active and healthy life in older patients with rhinitis and asthma across their life cycle. The app has proven to be very useful in managing allergic respiratory diseases in the elderly, with great appreciation from its users. To implement the adoption of the app across the territory, we have built a network of allergists throughout the entire Apulian region, in which each provincial capital is home for a main network node, coordinating and supporting nodes operating in smaller cities (63). The main Italian Scientific Society of Allergists (SIAAIC) has been involved in the project with the aim to organize meetings in order to spread the message and transfer the acquired know-how from allergic to chronic diseases.

\section{Acknowledgments}

Funding: None.

\section{Footnote}

Provenance and Peer Review: This article was commissioned by the Section Director (Yousser Mohammad, Alvaro A. Cruz) for the series "GARD Section" published in Fournal of Thoracic Disease. The article did not undergo external peer 
review.

Conflicts of Interest: All authors have completed the ICMJE uniform disclosure form (available at http://dx.doi. org/10.21037/jtd-21-792). The series GARD Section was commissioned by the editorial office without any funding or sponsorship. Dr. JB reports personal fees from Chiesi, Cipla, Hikma, Menarini, Mundipharma, Mylan, Novartis, Sanofi-Aventis, Takeda, Teva, Uriach, other from KYomedInnov, personal fees from Purina, outside the submitted work. Dr. JAF reports being a partner in a company developing mobile technologies for monitoring airways diseases. Dr. PK reports personal fees from Adamed, personal fees from Berlin Chemie Menarini, personal fees from Boehringer Ingelheim, personal fees from Chiesi, personal fees from Hal Allergy, personal fees from Lekam, personal fees from Novartis, personal fees from Polpharma, personal fees from Astra, outside the submitted work. Dr. RL reports grants and personal fees from GSK, grants and personal fees from $\mathrm{AZ}$, grants and personal fees from Novartis, grants from Chiesi, personal fees from Sanofi, outside the submitted work. Dr. TZ reports personal fees from Bayer Health Care, personal fees from FAES, personal fees from Novartis, personal fees from Henkel, from null, from null, from Novartis, from Henkel, personal fees from AstraZeneca Fee for talk, personal fees from AbbVie Fee for talk, personal fees from ALK Fee for talk, personal fees from Almirall Fee for talk, personal fees from Astellas Fee for talk, personal fees from Bayer Health Care Fee for talk, personal fees from Bencard Fee for talk, personal fees from Berlin Chemie Fee for talk, personal fees from FAES Fee for talk, personal fees from HAL Fee for talk, personal fees from Leti Fee for talk, personal fees from Meda Fee for talk, personal fees from Menarini Fee for talk, personal fees from Merck Fee for talk, personal fees from MSD Fee for talk, personal fees from Novartis Fee for talk, personal fees from Pfizer Fee for talk, personal fees from Sanofi Fee for talk, personal fees from Stallergenes Fee for talk, personal fees from Takeda Fee for talk, personal fees from Teva Fee for talk, personal fees from UCB Fee for talk, personal fees from Henkel Fee for talk, personal fees from Kryolan Fee for talk, personal fees from L'Oréal Fee for talk outside the submitted work. Dr. FSR reports personal fees from AstraZeneca, personal fees from Novartis, personal fees from Lusomedicamenta, personal fees from Sanofi, personal fees from GSK outside the submitted work. Dr. IT reports personal fees from Novartis, Boehringer Ingelheim, Astra Zeneca, GSK, grants from GSK Hellas, ELPEN, outside the submitted work. The authors have no other conflicts of interest to declare.

Ethical Statement: The authors are accountable for all aspects of the work in ensuring that questions related to the accuracy or integrity of any part of the work are appropriately investigated and resolved.

Open Access Statement: This is an Open Access article distributed in accordance with the Creative Commons Attribution-NonCommercial-NoDerivs 4.0 International License (CC BY-NC-ND 4.0), which permits the noncommercial replication and distribution of the article with the strict proviso that no changes or edits are made and the original work is properly cited (including links to both the formal publication through the relevant DOI and the license). See: https://creativecommons.org/licenses/by-nc-nd/4.0/.

\section{References}

1. Bousquet J, Arnavielhe S, Bedbrook A, et al. MASK 2017: ARIA digitally-enabled, integrated, person-centred care for rhinitis and asthma multimorbidity using real-worldevidence. Clin Transl Allergy 2018;8:45.

2. Bousquet J, Anto JM, Bachert C, et al. From ARIA guidelines to the digital transformation of health in rhinitis and asthma multimorbidity. Eur Respir J 2019;54:1901023.

3. Bousquet J, Bedbrook A, Czarlewski W, et al. Guidance to 2018 good practice: ARIA digitally-enabled, integrated, person-centred care for rhinitis and asthma. Clin Transl Allergy 2019;9:16.

4. Bousquet JJ, Schunemann HJ, Togias A, et al. Nextgeneration ARIA care pathways for rhinitis and asthma: a model for multimorbid chronic diseases. Clin Transl Allergy 2019;9:44.

5. Bousquet J, Anto JM, Bachert C, et al. ARIA digital anamorphosis: Digital transformation of health and care in airway diseases from research to practice. Allergy 2021;76:168-90.

6. Courbis AL, Murray RB, Arnavielhe S, et al. Electronic Clinical Decision Support System for allergic rhinitis management: MASK e-CDSS. Clin Exp Allergy 2018;48:1640-53.

7. Bousquet J, Agache I, Aliberti MR, et al. Transfer of innovation on allergic rhinitis and asthma multimorbidity in the elderly (MACVIA-ARIA) - EIP on AHA Twinning Reference Site (GARD research demonstration project). Allergy 2018;73:77-92. 
8. Bousquet J, Anto JM, Annesi-Maesano I, et al. POLLAR: Impact of air POLLution on Asthma and Rhinitis; a European Institute of Innovation and Technology Health (EIT Health) project. Clin Transl Allergy 2018;8:36.

9. Bédard A, Basagaña X, Anto JM, et al. Mobile technology offers novel insights into the control and treatment of allergic rhinitis: The MASK study. J Allergy Clin Immunol 2019;144:135-143.e6.

10. Bédard A, Sofiev M, Arnavielhe S, et al. Interactions Between Air Pollution and Pollen Season for Rhinitis Using Mobile Technology: A MASK-POLLAR Study. J Allergy Clin Immunol Pract 2020;8:1063-1073.e4.

11. Sofiev M, Palamarchuk Y, Bédard A, et al. A demonstration project of Global Alliance against Chronic Respiratory Diseases: Prediction of interactions between air pollution and allergen exposure-the Mobile Airways Sentinel NetworK-Impact of air POLLution on Asthma and Rhinitis approach. Chin Med J (Engl) 2020;133:1561-7.

12. Bousquet J, Anto JM, Haahtela T, et al. Digital transformation of health and care to sustain Planetary Health: The MASK proof-of-concept for airway diseasesPOLLAR symposium under the auspices of Finland's Presidency of the EU, 2019 and MACVIA-France, Global Alliance against Chronic Respiratory Diseases (GARD, WH0) demonstration project, Reference Site Collaborative Network of the European Innovation Partnership on Active and Healthy Ageing. Clin Transl Allergy 2020;10:24.

13. Mitsias DI, Dimou MV, Lakoumentas J, et al. Effect of nasal irrigation on allergic rhinitis control in children; complementarity between CARAT and MASK outcomes. Clin Transl Allergy 2020;10:9.

14. Sastre J, Del Cuvillo A, Colás C, et al. Validation of the MASK-air app for assessment of allergic rhinitis. Allergy 2020;75:2958-61.

15. Valiulis A, Bousquet J, Veryga A, et al. Vilnius Declaration on chronic respiratory diseases: multisectoral care pathways embedding guided self-management, mHealth and air pollution in chronic respiratory diseases. Clin Transl Allergy 2019;9:7.

16. Bousquet J, Farrell J, Illario M; ARIA-MASK study group. Aligning the Good Practice MASK With the Objectives of the European Innovation Partnership on Active and Healthy Ageing. Allergy Asthma Immunol Res 2020;12:238-58.

17. Bousquet J, Illario M, Farrell J, et al. The Reference Site Collaborative Network of the European Innovation Partnership on Active and Healthy Ageing. Transl Med
UniSa 2019;19:66-81.

18. Bousquet J, Pham-Thi N, Bedbrook A, et al. Nextgeneration care pathways for allergic rhinitis and asthma multimorbidity: a model for multimorbid noncommunicable diseases-Meeting Report (Part 2). J Thorac Dis 2019;11:4072-84.

19. Bousquet J, Pham-Thi N, Bedbrook A, et al. Nextgeneration care pathways for allergic rhinitis and asthma multimorbidity: a model for multimorbid noncommunicable diseases-Meeting Report (Part 1). J Thorac Dis 2019;11:3633-42.

20. Aguilar D, Lemonnier N, Koppelman GH, et al. Understanding allergic multimorbidity within the noneosinophilic interactome. PLoS One 2019;14:e0224448.

21. Lemonnier N, Melén E, Jiang Y, et al. A novel whole blood gene expression signature for asthma, dermatitis, and rhinitis multimorbidity in children and adolescents. Allergy 2020;75:3248-60.

22. Bousquet J, Devillier P, Anto JM, et al. Daily allergic multimorbidity in rhinitis using mobile technology: A novel concept of the MASK study. Allergy 2018;73:1622-31.

23. Amaral R, Bousquet J, Pereira AM, et al. Disentangling the heterogeneity of allergic respiratory diseases by latent class analysis reveals novel phenotypes. Allergy 2019;74:698-708.

24. Bousquet J, Anto JM, Wickman M, et al. Are allergic multimorbidities and $\mathrm{IgE}$ polysensitization associated with the persistence or re-occurrence of foetal type 2 signalling? The MeDALL hypothesis. Allergy 2015;70:1062-78.

25. Anto JM, Bousquet J, Akdis M, et al. Mechanisms of the Development of Allergy (MeDALL): Introducing novel concepts in allergy phenotypes. J Allergy Clin Immunol 2017;139:388-99.

26. Jiang Y, Gruzieva O, Wang T, et al. Transcriptomics of atopy and atopic asthma in white blood cells from children and adolescents. Eur Respir J 2019;53:1900102.

27. Patella V, Florio G, Magliacane D, et al. Public Prevention Plans to Manage Climate Change and Respiratory Allergic Diseases. Innovative Models Used in Campania Region (Italy): The Twinning Aria Implementation and the Allergy Safe Tree Decalogue. Transl Med UniSa 2019;19:95-102.

28. Cohen S, Kamarck T, Mermelstein R. A global measure of perceived stress. J Health Soc Behav 1983;24:385-96.

29. Menditto E, Costa E, Midao L, et al. Adherence to treatment in allergic rhinitis using mobile technology. The MASK Study. Clin Exp Allergy 2019;49:442-60.

30. Bousquet J, Mantzouranis E, Cruz AA, et al. Uniform 
definition of asthma severity, control, and exacerbations: document presented for the World Health Organization Consultation on Severe Asthma. J Allergy Clin Immunol 2010;126:926-38.

31. Barry MJ, Edgman-Levitan S. Shared decision making-pinnacle of patient-centered care. N Engl J Med 2012;366:780-1.

32. Abbasgholizadeh Rahimi S, Menear M, Robitaille H, et al. Are mobile health applications useful for supporting shared decision making in diagnostic and treatment decisions? Glob Health Action 2017;10:1332259.

33. Amaral R, Carneiro AC, Wandalsen G, et al. Control of Allergic Rhinitis and Asthma Test for Children (CARATKids): Validation in Brazil and cutoff values. Ann Allergy Asthma Immunol 2017;118:551-556.e2.

34. Fonseca JA, Nogueira-Silva L, Morais-Almeida M, et al. Validation of a questionnaire (CARAT10) to assess rhinitis and asthma in patients with asthma. Allergy 2010;65:1042-8.

35. Linhares DV, da Fonseca JA, Borrego LM, et al. Validation of control of allergic rhinitis and asthma test for children (CARATKids)--a prospective multicenter study. Pediatr Allergy Immunol 2014;25:173-9.

36. Samreth D, Arnavielhe S, Ingenrieth F, et al. Geolocation with respect to personal privacy for the Allergy Diary app - a MASK study. World Allergy Organ J 2018;11:15.

37. Laune D, Arnavielhe S, Viart F, et al. Adaptation of the General Data Protection Regulation (GDPR) to a smartphone app for rhinitis and asthma (MASK-airß). Rev Mal Respir 2019;36:1019-31.

38. Pereira AM, Jacome C, Almeida R, et al. How the Smartphone Is Changing Allergy Diagnostics. Curr Allergy Asthma Rep 2018;18:69.

39. Bousquet J, Hellings PW, Agache I, et al. Allergic Rhinitis and its Impact on Asthma (ARIA) Phase 4 (2018): Change management in allergic rhinitis and asthma multimorbidity using mobile technology. J Allergy Clin Immunol 2019;143:864-79.

40. Maurer M, Metz M, Bindslev-Jensen C, et al. Definition, aims, and implementation of GA(2)LEN Urticaria Centers of Reference and Excellence. Allergy 2016;71:1210-8.

41. Bousquet J, Burney PG, Zuberbier T, et al. GA2LEN (Global Allergy and Asthma European Network) addresses the allergy and asthma 'epidemic'. Allergy 2009;64:969-77.

42. Active Patients Access Care. 2020. Available online: https://www.efanet.org/campaigns/showleadership

43. Bonavita I, Rafael-Palou X, Ceresa M, et al. Integration of convolutional neural networks for pulmonary nodule malignancy assessment in a lung cancer classification pipeline. Comput Methods Programs Biomed 2020;185:105172.

44. Zimmer VA, Glocker B, Hahner N, et al. Learning and combining image neighborhoods using random forests for neonatal brain disease classification. Med Image Anal 2017;42:189-99.

45. Silver D, Schrittwieser J, Simonyan K, et al. Mastering the game of Go without human knowledge. Nature 2017;550:354-9.

46. Senna G, Guerriero M, Paggiaro PL, et al. SANI-Severe Asthma Network in Italy: a way forward to monitor severe asthma. Clin Mol Allergy 2017;15:9.

47. ISAR Study Group. International Severe Asthma Registry: Mission Statement. Chest 2020;157:805-14.

48. Djukanovic R, Adcock IM, Anderson G, et al. The Severe Heterogeneous Asthma Research collaboration, Patient-centred (SHARP) ERS Clinical Research Collaboration: a new dawn in asthma research. Eur Respir J 2018;52:1801671.

49. Canonica GW, Colombo GL, Bruno GM, et al. Shadow cost of oral corticosteroids-related adverse events: A pharmacoeconomic evaluation applied to real-life data from the Severe Asthma Network in Italy (SANI) registry. World Allergy Organ J 2019;12:100007.

50. Gissler M, Huakka J. Finnish health and social welfare registers in epidemiological research. Norsk Epidemiologi 2004;14:113-20.

51. Kilpeläinen K, Koponen P, Tolonen H, et al. From monitoring to action: utilising health survey data in national policy development and implementation in Finland. Arch Public Health 2019;77:48.

52. Wikström K, Toivakka M, Rautiainen P, et al. Electronic Health Records as Valuable Data Sources in the Health Care Quality Improvement Process. Health Serv Res Manag Epidemiol 2019;6:2333392819852879.

53. Lundqvist A, Mäki-Opas T. Health 2011 Survey Methods. The National Institute for Health and Welfare (THL), Report 8/2016, Helsinki, 2016. Available online: http://wwwjulkarifi/handle/10024/130780

54. Margaras V. Demographic Trends in the EU Region. 2019. Available online: https://ec.europa.eu/futurium/ en/system/files/ged/eprs-briefing-633160-demographictrends-eu-regions-final.pdf

55. State of Health Companion Report 2019. Available online: https://ec.europa.eu/health/sites/health/files/state/ docs/2019_companion_en.pdf

56. European Health Program 2020. Available online: https:// 
ec.europa.eu/health/funding/programme_en

57. Salas-Vega S, Haimann A, Mossialos E. Big Data and Health Care: Challenges and Opportunities for Coordinated Policy Development in the EU. Health Syst Reform 2015;1:285-300.

58. Shaping Europe's digital strategy. 2019. Available online: https://eceuropaeu/digital-single-market/en

59. EIP on AHA partnership marks. 2019. Available online: https://eceuropaeu/eip/ageing/design/logos_en

60. Bousquet J, Bewick M, Cano A, et al. Building Bridges for Innovation in Ageing: Synergies between Action Groups

Cite this article as: Bousquet J, Bedbrook A, Czarlewski W, De Carlo G, Fonseca JA, González Ballester MA, Illario M, Koskinen S, Laatikainen T, Onorato GL, Palkonen S, Patella V, Pham-Thi N, Puggioni F, Ventura MT, Joos G, Kuna P, Louis R, Makris M, Zalud P, Zuberbier T, Bachert C, Brussino L, Carreiro-Martins P, Carrion y Ribas C, Chalubinski M, Costa EM, de Vries G, Gemicioglu B, Gennimata D, Micheli Y, Niedoszytko M, Regateiro FS, Romantowski J, Taborda-Barata L, Toppila-Salmi S, Tsiligianni I, Viart F, Laune D. Digital Health Europe (DHE) Twinning on severe asthma-kick-off meeting report. J Thorac Dis 2021;13(5):3215-3225. doi: 10.21037/ jtd-21-792 of the EIP on AHA. J Nutr Health Aging 2017;21:92-104.

61. Blueprint for a digital transformation of health and care in an ageing society. 2018. Available online: https:// eceuropaeu/digital-single-market/en/blueprint-digitaltransformation-health-and-care-ageing-society

62. Dunn RM, Busse PJ, Wechsler ME. Asthma in the elderly and late-onset adult asthma. Allergy 2018;73:284-94.

63. Ventura MT, Gelardi M, D'Amato A, et al. Clinical and cytologic characteristics of allergic rhinitis in elderly patients. Ann Allergy Asthma Immunol 2012;108:141-4. 